\title{
Cone beam computed tomography - extending its arm to explore new possibilities
}

\author{
Prajakta P. Chaudhari ${ }^{A, B, C, D, E, F, G}$, Chetan Bhadage ${ }^{A, B, B, E, F}$, Ajay Bhoosreddy ${ }^{A, B, D, E}$, Pragati Bramhe ${ }^{A, D, E}$, \\ Prutha Rathod ${ }^{A, D, E}$, Shreya Dange ${ }^{A, B}$ \\ Department of Oral Medicine and Radiology, MGV's KBH Dental College and Hospital, Nashik, Maharashtra, India
}

\begin{abstract}
Purpose: To determine whether the greyscale value depicted in cone beam computed tomography (CBCT) differentiates different benign osseous lesions of jaws and to measure the greyscale value of various osseous lesions of jaws and to find the correlation (if any) of these greyscale values to that of histopathological diagnosis.

Material and methods: This study was conducted in the Department of Oral Medicine and Radiology of the Dental Institute after obtaining approval from the Ethical Committee. CBCT scans of osseous lesions of jaws confirmed with histopathological reports depicting cystic or tumour-like lesions were included in the study. Greyscale values depicted in CBCT scans of osseous lesions were measured. The greyscale values were grouped as per the histopathological diagnosis, and these ranges were then tabulated and statistically evaluated.
\end{abstract}

Results: The mean value with standard deviation of greyscale values for cystic lesions was $1208.375 \pm 93$ and for that of the tumour group was $1603 \pm 425.5$.

Conclusions: The greyscale value is a useful tool in differentiating between different groups of osseous lesions of jaws.

Key words: cyst, cone beam computed tomography, tumours, soft and hard tissue lesions, greyscale.

\section{Introduction}

Dental radiography plays an important role in the initial diagnosis and further treatment plan in routine dental practice. Usually orofacial pathologies are diagnosed on the basis of clinical, radiographic, and histopathological examination. Drawbacks arising in conventional 2D radiography can be offset by using three-dimensional imaging, i.e. cone beam computed tomography (CBCT). CBCT is a three-dimensional imaging modality that helps in the precise determination of the extent of lesions of orofacial structures and is quite helpful in the detection of odontogenic and non-odontogenic pathologies [1].

Recent CBCT systems with reduced radiation exposure, giving high-contrast images that are almost equal to bone window computed tomography (CT), have resulted in an increase in the indications for CBCT application in the evaluation of bone density assessment, complex diagnostic and treatment planning, surgical assessment of pathology, and much more [2,3].

Greyscale in CBCT is the ability of a scan to display differences in attenuation of radiation beam. This parameter is called the bit depth and it determines the number of shades of grey available to display the attenuation i.e. to detect the subtle contrast differences. The greyscale value or voxel value is the attenuation number of a particular matter, which is based on the density of the structure $[1,4]$. The use of CBCT as a delineating imaging modality for different osseous pathologies has been reported in very few studies [1,2,5-7].

Correspondence address:

Prajakta P. Chaudhari, Department of Oral Medicine and Radiology, MGV's KBH Dental College and Hospital, Mumbai Agra Road, Panchwati, Nashik,

Maharashtra, India, e-mail: drprajaktachaudhari@gmail.com

Authors' contribution:

A Study design · B Data collection · C Statistical analysis · D Data interpretation - E Manuscript preparation · F Literature search · G Funds collection 

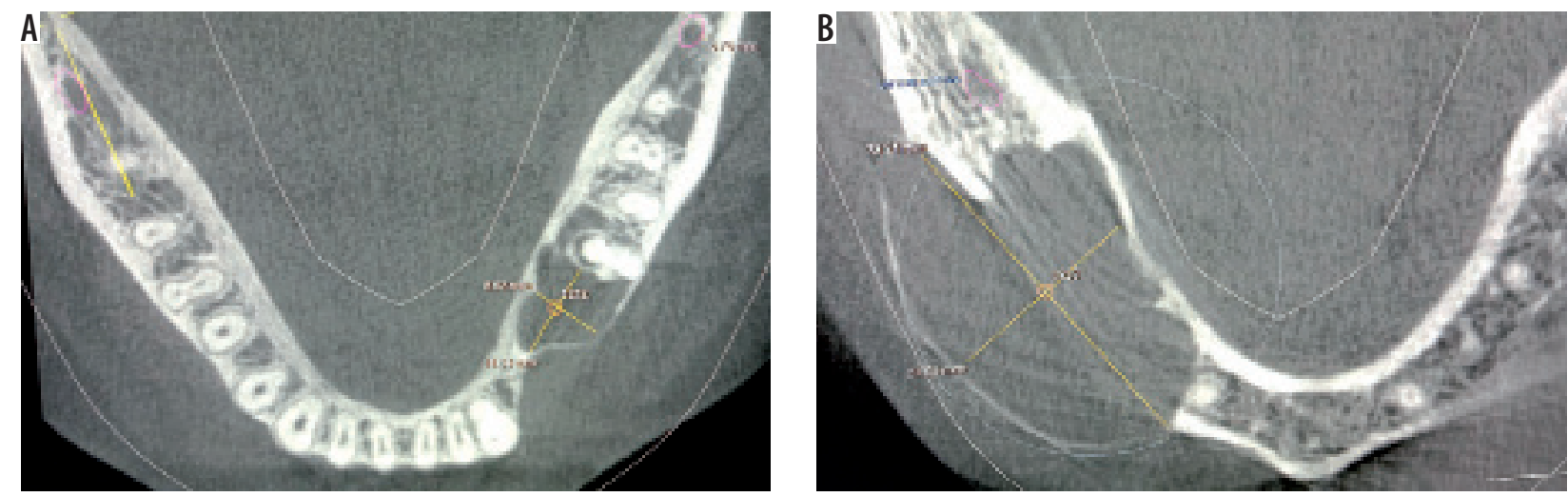

Figure 1. Cone beam computed tomography scan showing axial sections of two benign osseous lesions for the measurement of grey scale value at the epicentre of the lesion

Table 1. Age distribution

\begin{tabular}{|l|c|c|c|c|c|c|}
\hline Groups & $10-30$ years & $31-50$ years & $51-70$ years & 71 years and above & Mean & Total \\
\hline Cyst & $12(30.76 \%)$ & $17(43.58 \%)$ & $6(15.38 \%)$ & $4(10.2 \%)$ & 48.23 & 39 \\
\hline Tumour & $1(0.03 \%)$ & $11(33.33 \%)$ & $13(39.3 \%)$ & $8(24.2 \%)$ & 54.87 & 33 \\
\hline
\end{tabular}

Thus, the use of the greyscale value in assessing the internal tissue changes associated with osseous lesions of jaws can be of great help in posing the near final diagnosis or differentiation of different osseous lesions.

\section{Material and methods}

This was a retrospective study conducted in the Department of Oral Medicine and Radiology of the Dental Institute after obtaining the approval from the Ethical Committee. Previously available CBCT scans meeting the criteria were included in the study. Inclusion criteria included the following: CBCT scans of benign osseous lesions (cyst/tumour/tumour-like lesion) with confirmed histopathological reports. Exclusion criteria included the following: CBCT scans with blurred or distorted image and scans not accompanied by complete histopathological report.

A total of $72 \mathrm{CBCT}$ scans were evaluated using a SIRONA ORTHOPHOS XG 3D machine with $8 \times 8 \mathrm{~cm}$ field of view (FOV). While interpreting the CBCT scans the following routine radiographic parameters were examined for each lesions: location, periphery and shape, internal structure, and effect on surrounding structure.

Table 2. Site distribution

\begin{tabular}{|l|c|c|}
\hline Site & Cyst $(n=39)$ & Tumour $(n=33)$ \\
\hline Maxillary anterior & $12(30.76 \%)$ & $9(27.20 \%)$ \\
\hline Maxillary posterior & $7(17.94 \%)$ & $2(6.00 \%)$ \\
\hline Mandibular anterior & $6(15.38 \%)$ & $5(15.10 \%)$ \\
\hline Mandibular posterior & $9(23.07 \%)$ & $16(48.40 \%)$ \\
\hline Maxillary sinus & $5(12.82 \%)$ & $1(3.00 \%)$ \\
\hline
\end{tabular}

All the scans were divided into two groups based on the histopathological reports:

- group A - histopathological reports confirming cystic lesions $(n=39)$,

- group B - histopathological reports confirming tumour or tumour like lesions $(n=33)$.

The greyscale measurements were done on axial section of CBCT. For analysis of the grey values, the epicentre of the lesion was evaluated. The intersection of two perpendicular lines at the centre of the lesion was considered as the epicentre, as shown in Figure 1.

All the values were noted and tabulated to obtain the range for both the groups.

\section{Data analysis}

Data were analysed using Statistical Package for Social Sciences (SPSS) version 21, IBM Inc. Descriptive data were reported for each variable. Descriptive statistics such as mean and standard deviation for continuous variables and frequency along with percentages of categorical variables were calculated. A bivariate analysis was performed using independent $t$ test. The level of statistical significance was set at $p$ less than 0.05 .

\section{Results}

In this cross-sectional study, a total of $72 \mathrm{CBCT}$ scans along with the details showing osseous pathologies were obtained from the previous database. The age of the patients ranged from 10 to 84 years, with a mean age of 51.55 years (Table 1). Out of these 72 patients, 37 were male and 35 were female. In group A, 23 scans (58.97\%) were of males and 16 scans (41\%) were of females; the male to 
Table 3. Average greyscale values of group A (cystic lesions)

\begin{tabular}{|l|c|c|c|}
\hline Lesions & $n(\%)$ & Average greyscale value & Mean value with standard deviation \\
\hline Dentigerous cyst & $13(33.33)$ & $1197.0(1130-1248)$ & $1197.0 \pm 59.0$ \\
\hline Radicular cyst & $15(38.46)$ & $1209.5(1117-1258)$ & $1209.5 \pm 70.5$ \\
\hline Residual cyst & $3(7.69)$ & $1145.0(1130-1160)$ & $1145.0 \pm 15.0$ \\
\hline Odontogenic keratocyst & $8(20.51)$ & $1282.0(1267-1303)$ & $1282.0 \pm 18.0$ \\
\hline Total range & 39 & $1208.4(1117-1303)$ & $1208.4 \pm 93.0$ \\
\hline
\end{tabular}

Table 4. Average greyscale values for group B (tumour like lesions)

\begin{tabular}{|l|c|c|c|}
\hline Lesions & $n(\%)$ & Average greyscale value & Mean value with standard deviation \\
\hline Ameloblastoma & $10(30.30)$ & $1369.33(1312-1437)$ & $1369.3 \pm 62.5$ \\
\hline Central giant-cell granuloma & $3(9.09)$ & $1699.00(1664-1734)$ & $1699.0 \pm 35.0$ \\
\hline Central ossifying fibroma & $2(6.06)$ & 1417 & 1417 \\
\hline Adenomatoid odontogenic tumour & $12(36.36)$ & $1391.66(1303-1441)$ & $1391.6 \pm 64.0$ \\
\hline Odontome & $6(18.18)$ & $2138.50(2123-2154)$ & $2138.5 \pm 15.5$ \\
\hline Total range & 33 & $1603.10(1303-2154)$ & $1603.0 \pm 425.5$ \\
\hline
\end{tabular}

Table 5. Comparison between greyscale values of cysts and tumours

\begin{tabular}{|l|c|c|c|c|c|c|}
\hline Groups & Average greyscale value & Standard deviation & SE & $t$ value & $p$ value & Remark \\
\cline { 1 - 5 } Cyst & 1208.375 & 93.0 & 75.5522 & -5.6416 & 0.0001 & Significant \\
\cline { 1 - 3 } & 1603.000 & 425.5 & & & & \\
\hline
\end{tabular}

Table 6. Greyscale values for different types of lesions

\begin{tabular}{|l|c|c|c|c|}
\hline Groups & $\begin{array}{c}\text { Average } \\
\text { greyscale } \\
\text { value }\end{array}$ & $\begin{array}{c}\text { Radiolucent } \\
(n=41)\end{array}$ & $\begin{array}{c}\text { Mixed } \\
(n=25)\end{array}$ & $\begin{array}{c}\text { Radiopaque } \\
(n=6)\end{array}$ \\
\hline Cyst & 1208.375 & $1117-1290$ & $1214-1303$ & - \\
\hline Tumour & 1603.098 & $1303-1359$ & $1312-1734$ & $2123-2154$ \\
\hline
\end{tabular}

female ratio was $1.4: 1$. In group B the ratio was $1: 1.3$, in which $14(42.4 \%)$ scans were of males and 19 (57.6\%) scans were of females. The detailed distribution of the patients and the lesion's locations are mentioned in Table 2.

In group A, increased predilection was seen in the case of the maxillary anterior region, at $30.76 \%$, and in group $B$ the posterior mandible, at $48.4 \%$, was seen to be a more common site. The least occurrence was seen with maxillary sinus followed by maxillary posteriors in both groups.

Bone density was calculated by the greyscale measurements taken from the epicentre of each lesion in group A and group B were noted and tabulated to find out the mean range. The maximum average greyscale value was noted for odontogenic keratocyst, with the mean value and standard deviation of $1282 \pm 18$, while the lowest range of greyscale value was seen for residual cyst, with the mean and standard deviation of $1145 \pm 15$ (Table 3).
Table 4 shows the greyscale values of group B, which included the tumour-like lesions; it was found that it showed a higher range of greyscale values with the maximum being the odontome, with the mean and standard deviation being $2138.5 \pm 15.5$. The mean value with standard deviation for the greyscale values of ameloblastoma and adenomatoid odontogenic tumour were $1369.33 \pm 62.5$ and $1391.66 \pm 64.0$, respectively.

The total range for the greyscale values for cyst was 1117-1303 with the mean value and standard deviation being $1208.375 \pm 93$, whereas the total range for the greyscale values for tumour was 1117-1303, with the mean value and standard deviation being $1208.375 \pm 93$.

There was a clear difference in the ranges of the mean greyscale values for cysts and tumours.

Unpaired $t$ test was applied to check if the difference in average greyscale values was significant. The results obtained are shown in Table 5.

It can be concluded from the above table that there was a significant difference between the average greyscale values of cystic lesions and tumour lesions. This feature may be used to identify the type of lesion, because it is clear from the above-average greyscale values that these values are higher in the case of tumour lesions than those of cystic lesions. 
Table 6 shows the different ranges greyscale values in radiopaque, radiolucent, and mixed lesions in cysts and tumour-like lesions.

\section{Discussion}

With the advent of radiology, multiple studies have been carried out for the diagnosis and differentiation between different pathologies of the jaw. Odontogenic pathologies closely resemble each other, making it more difficult to differentiate them clinically and radiographically. Since three-dimensional imaging modalities like computed tomography scan (CT scan), magnetic resonance imaging (MRI), and CBCT came into the picture there has been a tremendous increase in the advantages associated with diagnosis and treatment planning. The radiation attenuation in $\mathrm{CT}$ imaging of different tissue is calculated in Hounsfield units (HU) thus making it easier to define what a given finding may represent. HU measures the radiodensity on a quantitative scale $[1,8]$. CBCT introduced for dental use has many advantages such as high resolution acquisition and, compared with conventional CT, lower radiation doses with smaller and less expensive equipment [9]. A large number of studies have shown a linear relationship between Hounsfield units (HU) in CT scans and greyscale in CBCT, which can be used for the estimation of bone density $[8,10]$.

Patrick et al. [11] in the year 2017 evaluated the correlation between Hounsfield units of multislice computed tomography (MSCT) scans and greyscale values in CBCT in various fields of view for hyperdense as well as hypodense structures. It was found that the grey values for hypodense structures in large-volume CBCT scans were more reliable and analogous to Hounsfield units in MSCT. However, for hyperdense structures the grey value of CBCT may not be as accurate as the $\mathrm{HU}$ value of a CT scan.

In limited volume CBCT imaging, the attenuation of X-ray beam becomes maximised and minimised at various points in the scan due to the $360^{\circ}$ rotation of the CBCT machine for the data acquisition. This phenomenon might in turn reduce the overall density value in the limited imaging volume [8].

Also, the use of anti-scatter grids in CT scans helps to reduce the scattered radiation which cannot be prevented in CBCT. This causes an increased resultant beam hardening effect, partial volume averaging, and under sampling of the images in CBCT scans, due to which a few authors like Armstrong et al. [12] and Miles et al. [13] suggested a negative correlation between Hounsfield units of CT scans and greyscale values of CBCT.

So, in the present study we tried to use the greyscale value for the detection and differentiation of different osseous lesions.
Trope et al. [14] carried out an in-vitro study by subjecting mandibles of human cadavers to CT scan examinations and found that a cyst could be differentiated from a granuloma by this method. A similar in-vivo study was carried out by Juerchott et al. [15] in the year 2018, in which MRI was found to be useful in the differentiation between cyst and granuloma. Mishra et al. [2] reported in their study that, when taking into consideration the exposure parameters and cost, CBCT may be more convenient and suitable than multislice computed tomography for the evaluation of bony lesions of the jaws caused by cysts and tumours.

Greyscale value for determination of the lesion was also carried out on digitalised radiograph by Shrout et al. [5], who in their study compared the greyscale values of cysts and granulomas on a histogram. It was concluded that granulomas had a narrower range and lower greyscale value than cyst.

Results obtained in the study given by Nasim et al. [1] also showed that there is strong correlation of greyscale value that is seen in cases of cysts and tumours which can be attributed to the bone density level and therefore considered as a major diagnostic tool in the branch of dentistry.

The present study was aimed in determining whether the greyscale values depicted in CBCT differentiate different benign osseous lesions of the jaw. Hence, the greyscale values were calculated for the geometric centres of the lesion. In most instances the geometric centre of the lesion contains tissue that is homogenous, i.e. it contains either solid mass of tissue or fluid or air, etc.

Because determining the greyscale value for borders or margins of the lesion was beyond the scope of our study, the greyscale values were not calculated at the margins. We were not able to find any literature addressing the greyscale values for the borders of the lesion, and thus an entirely new study should be planned to assess the greyscale values for the lesion margins and borders.

In the present study, a definitive difference in the ranges of greyscale values between cysts and tumour lesions was noted, with the tumours having a higher range of values when compared to cysts. Thus, the greyscale value can be used as one of the differentiating aids in benign osseous lesion of the jaw.

\section{Conclusions}

Within the scope of our study, we can conclude that the greyscale value of CBCT can be used for the diagnosis of benign osseous jaw lesions. However, further studies are suggested with different CBCT scanners for greater accuracy.

\section{Conflict of interest}

The authors report no conflict of interest. 


\section{References}

1. Nasim A, Sasankoti Mohan RP, et al. Application of cone beam computed tomography grey scale values in the diagnosis of cysts and tumours. J Indian Acad Oral Med Radiol 2018; 30: 4-9.

2. Mishra SS, Degwekar SS, Banode PJ, et al. Comparative study of cone beam computed tomography and multislice computed tomography in the radiographic evaluation of cyst and tumours of the jaw. J Indian Acad Oral Med Radiol 2014; 26: 253259.

3. Schulze D, Blessmann M, Pohlenz P, et al. Diagnostic criteria for the detection of mandibular osteomyelitis using cone-beam computed tomography. Dentomaxillofac Radiol 2006; 35: 232-235.

4. Scarfe WC, Farman AG. Cone beam computed tomography; volume acquisition. In: White SC, Pharoah MJ (eds.). Oral Radiology: Principle and Interpretation. $7^{\text {th }}$ ed. Mosby; 2014. p. 185200.

5. Shrout MK, Hall JM, Hildebolt CE, et al. Differentiation of periapical granuloma and radicular cyst by digital radiometric analysis. Oral Surg Oral Med Oral Pathol 1993; 76: 356361.

6. Simon HS, Enciso R, Malfaz JM, et al. Differential diagnosis of large periapical lesion using cone beam computed tomography measurement and biopsy. J Endod 2006; 32: 833837.

7. Camps J, Pommel L, Bukiet F, et al. Evaluation of periapical lesion healing by correction of grey values. J Endod 2004; 30: 762766.

8. Razi T, Niknami M, Alavi Ghazani F, et al. Relationship between Hounsfield unit in CT scan and grey scale in CBCT. J Dent Res Dent Clin Dent Prospects 2014; 8: 107110.
9. White SC, Pharoah MJ. Oral Radiology: Principles and Interpretation. $6^{\text {th }}$ ed. St Louis: Mosby; 2009. p. 235236.

10. Mah P, Reeves TE, McDavid WD, et al. Deriving Hounsfield units using grey levels in cone beam computed tomography. Dentomaxillofac Radiol 2010; 39: 323335.

11. Patrick S, Birur NP, Gurushanth K, Shubhasini Raghavan A, Gurudath $S$. Comparison of grey values of cone-beam computed tomography with Hounsfield units of multislice computed tomography: an in vitro study. Indian J Dent Res 2017; 28: 66-70.

12. Armstrong RT. Acceptability of cone beam CT vs. multi-detector CT for 3D anatomic model construction. J Oral Maxillofac Surg 2006; 64: 37.

13. Miles DA, Danforth RA. A clinician's guide to understanding cone beam volumetric imaging (CBVI). Peer Reviewed Publ Acad Dent Ther Stomatol 2008; 1: 2-13.

14. Trope M, Pettigrew J, Petras J, et al. Differentiation of radicular cyst and granulomas using computerized tomography. Endod Dent Traumatol 1989; 5: 69-72.

15. Juerchott A, Pfefferle T, Flechtenmacher C, et al. Differentiation of periapical granulomas and cysts by using dental MRI: a pilot study. Int J Oral Sci 2018; 10: 17. 